substituted benzenes. This also confirms the results of our calculations. The details will be published elsewhere.

The writer wishes to express his sincere thanks to Professor S. Mizushima and Dr. T. Shimanouchi at Tokyo University for their kind guidance.

1 Young. Duvall, and Wright, Anal. Chem. 23, 709 (1951).

Y. Kakiuti and T. Shimanouchi, J. Chem. Phys. (to be published). Shimanouchi, Kakiuti, and Gamo, J. Chem. Phys, (to be published).

4 American Petroleum Institute, Research Project 44, catalog of infrared and Raman spectra.

5 D. H. Whiffen, Spectrochim. Acta 7, 253 (1955).

\section{Assignment of the OH In-Plane Deformation Frequency}

S. Krimm, C. Y. Liang,* and G. B. B. M. Sutherland $\dagger$ Harrison M. Randall Laboratory of Physics, University of Michigan, Ann Arbor, Michigan

(Received August 3, 1956)

$T^{\mathrm{H}}$ $\mathrm{HE}$ assignment of the $\mathrm{OH}$ in-plane deformation frequency in alcohols, $\delta(\mathrm{OH})$, in which the hydrogen atom moves in the $\mathrm{COH}$ plane and essentially perpendicular to the $\mathrm{OH}$ bond, has been controversial for some time. ${ }^{1}$ On the basis of a study of the spectrum of methyl alcohol, some authors ${ }^{2,3}$ have favored a value near $1110 \mathrm{~cm}^{-1}$ for this vibration, whereas others $\mathrm{s}^{4,5}$ have assigned it to a band at $1340 \mathrm{~cm}^{-1}$. It has also been suggested that this vibration occurs in the 1050 to $1080 \mathrm{~cm}^{-1}$ region,,$^{6,7}$ and near $1020 \mathrm{~cm}^{-1.8}$

In a recent detailed study of a series of alcohols, Stuart and Sutherland ${ }^{9}$ found that on dilution a broad doublet with peaks at 1330 and $1410 \mathrm{~cm}^{-1}$ was replaced by a single band near $1250 \mathrm{~cm}^{-1}$, and that a band near $1110 \mathrm{~cm}^{-1}$ shifted to about $1080 \mathrm{~cm}^{-1}$. On deuteration, a band appeared near $950 \mathrm{~cm}^{-1}$ which shifted to 900 $\mathrm{cm}^{-1}$ on dilution. They concluded from these and other results that the $\delta(\mathrm{OH})$ vibration in the associated alcohols should be assigned to the 1410 to $1330 \mathrm{~cm}^{-1}$ doublet, although no satisfactory explanation could be given for the doublet structure of this band. We wish to report some results of a study of the spectrum of polyvinyl alcohol ${ }^{10}$ which bear on the assignment of the $\delta(\mathrm{OH})$ frequency.

We have obtained the infrared spectra of normal and of deuterated polyvinyl alcohol, ${ }^{10,11}$ The significant changes on deuteration are the disappearance of bands at $1446 \mathrm{~cm}^{-1}, 1326$ $\mathrm{cm}^{-1}$, and $1096 \mathrm{~cm}^{-1}$, and the appearance of bands at $1384 \mathrm{~cm}^{-1}$, $1052 \mathrm{~cm}^{-1}$, and $985 \mathrm{~cm}^{-1}$, all of these bands being of comparable intensity. It seems fairly certain ${ }^{10}$ that the $1096-\mathrm{cm}^{-1}$ band, and its counterpart at $1052 \mathrm{~cm}^{-1}$, is related to a $\nu(\mathrm{CO})$ vibration, and that the $985 \mathrm{~cm}^{-1}$ band is a $\delta(O D)$ vibration. There is also good evidence $^{10,12}$ that the $1384-\mathrm{cm}^{-1}$ band is to be assigned to a $\mathrm{CH}$ bending mode, $\delta(\mathrm{CH})$. We therefore suggest that the $\delta(\mathrm{OH})$ mode of the associated molecule interacts with the $\delta(\mathrm{CH})$ mode, giving rise to two mixed vibrations $\delta(\mathrm{OH}+\mathrm{CH})$, whose frequencies are close to $1330 \mathrm{~cm}^{-1}$ and $1410 \mathrm{~cm}^{-1}$ (or $1446 \mathrm{~cm}^{-1}$ in polyvinyl alcohol). In the monomeric state of the alcohols this interaction disappears, leaving a single $\delta(\mathrm{OH})$ frequency at about $1250 \mathrm{~cm}^{-1}$. The same happens on deuteration, giving a single $\delta(O D)$ frequency at about $950 \mathrm{~cm}^{-1}$.

If the foregoing explanation is correct, we would expect that upon dilution of a secondary alcohol a band near $1380 \mathrm{~cm}^{-1}$ should become more intense, representing the uncoupled $\delta(\mathrm{CH})$ mode. This has indeed been found ${ }^{13}$ in dilution studies on pentanol-3. Similarly, we would predict that the $\delta(\mathrm{OH})$ frequency of associated tertiary alcohols should be single, and this again is in accord with the observations. ${ }^{9}$ The experimental evidence is less clear in the case of primary alcohols, where we would expect the doubling to arise from coupling between the $\delta(\mathrm{OH})$ and $\delta\left(\mathrm{CH}_{2}\right)$ vibrations. However, there do seem to be some apparently anomalous frequency and intensity changes in this region on dilution. ${ }^{9}$ The situation in polyvinyl alcohol is more clear-cut because of the absence of $\mathrm{CH}_{3}$ group absorption, and because of simplifications introduced by the repeating chain. In order to clarify the problem in the case of the primary alcohols, more observations are needed on alcohols in which the $\mathrm{CH}_{2}$ and $\mathrm{OH}$ groups have been deuterated, both individually and simultaneously.

* Present address: American Viscose Corporation, Marcus Hook, Pennsylvania.

$\dagger$ Present address: National Physical Laboratory, Teddington, Middlesex, England

G. B. B. M. Sutherland, Discussions Faraday Soc. 9, 274 (1950).

2 A. Borden and E. F. Barker, J. Chem. Phys. 6, 553 (1938)

3 M. Davies, J. Chem. Phys. 16, 267 (1948).

1 H. D. Noether. J. Chem. Phys. 10, 693 (1942).

${ }^{5} \mathrm{G}$. Herzberg, Infrared and Raman Spectra (D. Van Nostrand Company. Inc., New York, 1945), p. 334.

6 Barnes. Gore, Stafford, and Williams, Anal. Chem. 20, 402 (1948).

7 H. W. Thompson, J. Chem. Soc. 328 (1948).

8 J. R. Quinan and S. E. Wiberley, J. Chem. Phys. 21, 1896 (1953); Anal. Chem. 26, 1762 (1954)

${ }^{9}$ A. V. Stuart and G. B. B. M. Sutherland, J. Chem. Phys, 24, 559 (1956).

10 Krimm, Liang, and Sutherland, Symposium on Molecular Structure and Spectroscopy, Columbus, Ohio, June, 1956; J. Polymer Sci. (to be published).

11 Tadokoro, Seki, and Nitta, J. Chem. Phys. 23, 1351 (1955).

12 S. Krimm and C. Y. Liang, J. Polymer Sci. (to be published) 18 F. A. Smith and E. C. Creitz, J. Research Natl. Bur. Standards 46, 145
(1951).

\author{
Millimeter-Wave Lines of Heavy Water* \\ Gunnar Erlandsson and John Cox \\ Department of Physics, Duke University, Durham, North Carolina \\ (Received August 13, 1956)
}

$F^{\mathrm{r}}$

ROM the rotational and centrifugal distortion constants of HDO given by Posener ${ }^{1,2}$ the $1_{1,1} \rightarrow 1_{1,0}$ line of HDO is predicted to $80606 \mathrm{Mc}$. The constants given by Weisbaum et al. ${ }^{3.4}$ give $80545 \mathrm{Mc}$ for the same line. The corresponding predictions for the $2_{1,2} \rightarrow 2_{1,1}$ line are $241659 \mathrm{Mc}$ and $241436 \mathrm{Mc}$, respectively. These lines have been observed here at frequencies, which are about intermediate between the two predictions, namely 80578.15 $\mathrm{Mc}$ and $241561.3 \mathrm{Mc}$. The first line has been identified by means of its Stark effect whereas the identification of the latter is still a matter of probability.

Since the rigid rotor frequency of the $2_{1}$ line is exactly three times that of the $1_{1}$ line, this pair gives directly some information about the centrifugal distortion. Neglecting the $(K, K \pm 1)$ off diagonal elements in the energy matrix one gets

$$
\left\{\begin{array}{l}
\nu\left(1_{1}\right)=\nu_{0}\left(1_{1}\right)-8 \delta_{J}+8 R_{b} \\
\nu\left(2_{1}\right)=3 \nu_{0}\left(1_{1}\right)-72 \delta_{J}+24 R_{b}
\end{array}\right.
$$

where $\nu_{0}$ is the rigid rotor frequency. (The same relations may be derived from the Kivelson-Wilson ${ }^{5}$ approximation formula for $Q$-branch lines.) Eliminating $\nu_{0}+8 R_{5}$ one obtains $\delta_{J}=3.61 \pm 0.04$ Mc, which is somewhat closer to Posener's theoretical value 3.333 Mc than to Weisbaum's empirical 4.168 Mc.

In addition to the above lines a number of other lines of HDO and $\mathrm{D}_{2} \mathrm{O}$ have been observed in the millimeter region. The identification of these has been greatly facilitated by the infrared results of Benedict et al. ${ }^{6} \mathrm{In}$ two cases (HDO and $\mathrm{D}_{2} \mathrm{O}, 5_{2,3} \rightarrow 6_{1,6}$ ) the lines were found as a result of searching at the frequencies predicted by them ( 138.5 and $90.5 \mathrm{kMc}$ ). The observed lines and their assignments are given in Table I. The somewhat tedious task

TABLE I.

\begin{tabular}{|c|c|c|}
\hline Molecule & Transition & $\begin{array}{c}\text { Frequency } \\
\text { Mc }\end{array}$ \\
\hline $\mathrm{HDO}$ & $\begin{array}{l}1_{1,1} \rightarrow 11,0 \\
2_{1,2} \rightarrow 21,1 \\
3_{2,2} \rightarrow 32,1 \\
4_{2,3} \rightarrow 42,2 \\
4_{2,2} \rightarrow 5_{1,5} \\
5_{2,8} \rightarrow 61,6\end{array}$ & $\begin{array}{rl}80 & 578.15 \\
241 & 561.3 \\
50 & 236.30 \\
143 & 727.2 \\
120 & 778.2 \\
138 & 530.4\end{array}$ \\
\hline $\mathrm{D}_{2} \mathrm{O}$ & $\begin{array}{l}3_{3,0} \rightarrow 42,3 \\
5_{3,3} \rightarrow 44,0 \\
5_{2,3} \rightarrow 6_{1,6}\end{array}$ & $\begin{array}{l}43414.57 \\
55482.32 \\
90916.8\end{array}$ \\
\hline
\end{tabular}

a Reported at 50236.90 by Strandberg. J. Chem. Phys. 17, 901 (1949). 
of refining the constants for HDO to fit the new data has hitherto only been started. It is hoped that it can be put up to an electronic computer later on.

We are grateful to Professor Walter Gordy for suggesting this work and for helpful discussions.

* This research was supported by the U. S. Air Force through the Office of Scientific Research of the Air Research and Development Command

under Contract No. AF18(600)-497.
i D. W. Posener, Massachusetts Institute of Technology Technical Report No. 255 (1953).

2D. W. Posener and M. W. P. Strandberg. J. Chem. Phys. 21, 1401 (1953).

${ }^{3} \mathrm{~S}$. Weisbaum and Y. Beers, J. Chem. Phys. 23, 1606 (1955).

4 Beers, Weisbaum, Herrmann, and Sterzer, New York University College of Engineering Report, No. 195.2 (1953).

D. Kivelson and E. B. Wilson, Jr., J. Chem. Phys, 20, 1575 (1952).

- Benedict, Gailar, and Plyler, J. Chem. Phys. 24, 1139 (1956).

\section{Dipole Moment of $\mathbf{N F}_{3}$ \\ PaUl Kisliuk \\ Bell Telephone Laboratories, Murray Hill, New Jersey \\ (Received July 6, 1956)}

$I^{\mathrm{N}}$ a recent letter, ${ }^{1}$ Mashima presented calculations intended to show that the dipole moment of $\mathrm{NF}_{3}$ can be satisfactorily explained assuming directed single bonds with the amount of $s-p$ hybridization of the $N$ orbitals required by the observed bond angle. The present author has contended ${ }^{2}$ that the quadrupole coupling of the $N$ nucleus in $\mathrm{NF}_{3}$ can be reasonably explained only by abandoning the directed single bond model and adopting a multiple bonded structure in which the $s-p$ hybridization is not simply related to the bond angle.

In his calculation Mashima has ignored the possible ionic character of the $\mathrm{N}-\mathrm{F}$ bond. Using a recently published table, ${ }^{3}$ the electronegativity difference $X_{\mathrm{F}}-X_{\mathrm{N}}$ is 0.95 . This would correspond, according to estimates by various authors, ${ }^{4}$ to between $42 \%$ and $51 \%$ ionic character for a corresponding diatomic molecule, so that one might estimate each $\mathrm{N}-\mathrm{F}$ bond in $\mathrm{NF}_{3}$ to be roughly $16 \%$ ionic. This is sufficient to severely influence a calculation of the dipole moment.

Evidence of partial multiple bonding arises from the observation that the $\mathrm{N}-\mathrm{F}$ bond distance in $\mathrm{NF}_{3}(1.37 \mathrm{~A})^{5}$ is significantly less than that in $\mathrm{N}_{2} \mathrm{~F}_{2}(1.46 \mathrm{~A}){ }^{6}$ Inclusion of partial multiple bonding would presumably affect the computed bond angle and dipole moment.

Even if one accepts a model without partial ionic or multiple character, agreement with the observed dipole moment is obtained in Mashima's calculation only for an unusually large $s$ hybridization of the $F$ orbitals, i.e., $\sim 53 \%$. This is more than is required for maximum overlap, i.e., about $25 \%,{ }^{7}$ and much more than is generally attributed to halogen bonding orbitals. ${ }^{7-9}$

Unfortunately evidence relating to the type of bonding from the nuclear quadrupole coupling of nitrogen presents considerable difficulty in interpretation, ${ }^{2,10}$ so that speculation on this basis as to the bonding orbitals in $\mathrm{NF}_{3}$ may be fruitless.

Calculations on the basis of such a simple model as that used by Mashima, showing that the observed bond angle and dipole moment can be obtained by a suitable choice of two adjustable parameters, would be of enhanced interest if further understanding of the bond energy, bond length, or nuclear quadrupole coupling followed from the orbitals so obtained.

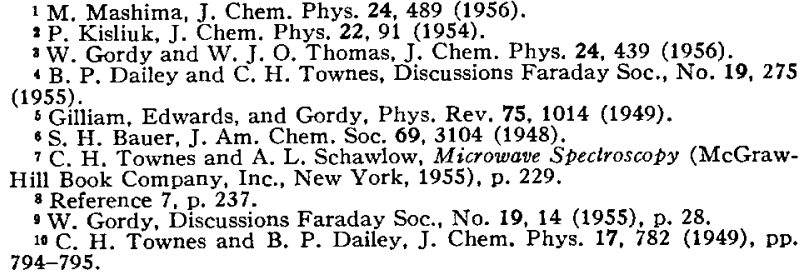

\section{Dipole Moment of $\mathrm{NF}_{3}$}

M. Mashima

Liberal Arts Faculty, Saga University, Saga-City, Japan

(Received July 26, 1956)

I $\mathrm{T}$ is pertinent to add to the Letter of $\mathrm{P}$. Kisliuk, ${ }^{1}$ under the 1 above title, the following remarks. In a previous Letter, ${ }^{2}$ the author has indicated that the observed dipole moment of $\mathrm{NF}_{3}$ can be satisfactorily explained assuming $s-p$ hybridization of the $N$ and $\mathrm{F}$ parts of the bonding wave function.

Kisliuk ${ }^{1}$ points out that the $s$ hybridization (53\%) of the $\mathrm{F}$ bond orbital is unusually large. According to the author's calculation, $53.4 \% s$ character is required for maximum overlap, and agreement with the observed dipole moment is obtained for $51.5 \%$ or $55.8 \%$ $s$ character. Thus, it may be said that the principle of maximum overlapping is nearly satisfied.

For another example, $\mathrm{PCl}_{3}$, we can see that maximum overlap is attained at $45.2 \% s$ character of the $\mathrm{Cl}$ bond orbital, and agreement with the observed dipole moment is obtained at $40.5 \%$ $s$ character. It is clear that similar character of the bond orbitals in these molecules is reasonable, since the electronegativity ${ }^{3}$ differences in $\mathrm{NF}_{3}$ and $\mathrm{PCl}_{3}$ are 0.95 and 0.9 , respectively.

For $\mathrm{PF}_{3}$ (with the electronegativity difference $X_{\mathrm{F}}-X_{\mathrm{P}}=1.85$ ), there is a striking difference in that the observed dipole moment is larger than the maximum value calculated by the same type of assumption as $\mathrm{NF}_{3}$. Thus, the observed dipole moment cannot be explained merely in terms of hybridization, but the ionic terms must be added to the bonding wave function.

It is of interest to see that with regard to $\mathrm{HCl}$ (with $X_{\mathrm{Cl}}-X_{\mathrm{H}}$ $=0.9)$ and $\mathrm{HF}\left(X_{\mathrm{F}}-X_{\mathrm{H}}=1.4\right)$, similar circumstances have been indicated by Warhurst and Whittle." Of course, further checks of the simple model with experiment are needed before it may be used with confidence.

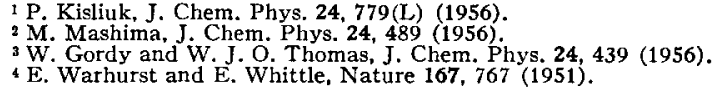

\section{Electrostatic Interpretation of Directed Valence}

\author{
B. F. Gray and H. O. Pritchard \\ Department of Chemistry, University of Manchester. \\ Manchester 13, England \\ (Received July 19, 1956)
}

GINCE the early 1930 's, it has been common to discuss bond angles in terms of directional properties of atomic orbitals. It is clear, however, that there is no causal relationship between the mathematical convenience of the orbital approximation and the occurrence of directed valences. We will take the two molecules $\mathrm{BeH}_{2}$ and $\mathrm{H}_{2} \mathrm{O}$ as typifying the problem of directed valence and show that their difference in shape can be understood qualitatively in terms of an electrostatic model.

First, consider the symmetrical approach of two protons $P$ to a hydrogen-like atom $M$ : the energy of repulsion between the three nuclei will be a minimum when they are in a straight line $P-M-P$, but the energy of altraction between the electron and the nucle $i$ is a maximum when the two $P$ nuclei are superimposed, i.e., $[2 P]-M$ (compare the $\mathrm{H}_{3}{ }^{++}$molecule-ion, where approximate electronic attraction energies for $4 a_{0}$ separation are $1,2: \theta=180^{\circ}$, $\left.E \approx-2.6 I_{H} ; \theta=60^{\circ}, E \approx-3.3 I_{H} ; \theta=0, E \approx-4.5 I_{H}\right)$. For any given $M-P$ distance, the expected bond angle will depend on the absolute magnitudes of these two energy terms and the way in which they vary with the $P-M-P$ angle. The equilibrium molecular configuration arises from a compromise between these considerations and the way in which the two energy terms vary with internuclear separation.

The compounds $M H_{n}$ may be divided into two classes, (1) where the nuclear repulsion term dominates and (2) where the net electronic attraction term dominates. Consider the approach of 